Virginia Commonwealth University

VCU Scholars Compass

1998

\title{
Dynamics and instabilities near the glass transition: From clusters to crystals
}

\author{
Saroj K. Nayak \\ Virginia Commonwealth University \\ Puru Jena \\ Virginia Commonwealth University, pjena@vcu.edu \\ Keith D. Ball \\ University of Chicago \\ R. Stephen Berry \\ University of Chicago
}

Follow this and additional works at: http://scholarscompass.vcu.edu/phys_pubs

Part of the Physics Commons

Nayak, S. K., Jena, P., \& Ball, K. D., et al. Dynamics and instabilities near the glass transition: From clusters to crystals. The Journal of Chemical Physics, 108, 234 (1998). Copyright (C) 1998 American Institute of Physics.

\section{Downloaded from}

http://scholarscompass.vcu.edu/phys_pubs/142

This Article is brought to you for free and open access by the Dept. of Physics at VCU Scholars Compass. It has been accepted for inclusion in Physics Publications by an authorized administrator of VCU Scholars Compass. For more information, please contact libcompass@vcu.edu. 


\title{
Dynamics and instabilities near the glass transition: From clusters to crystals
}

\author{
Saroj K. Nayak and Puru Jena \\ Physics Department, Virginia Commonwealth University, Richmond, Virginia 23284 \\ Keith D. Ball \\ Department of Physics, The University of Chicago, Chicago, Illinois 60637 \\ R. Stephen Berry \\ Department of Chemistry, The University of Chicago, Chicago, Illinois 60637
}

(Received 18 August 1997; accepted 26 September 1997)

\begin{abstract}
Molecular dynamics simulation has been used to explore the evolution, kinetics, and dynamics of a liquid-glass transition in clusters and bulk matter. We demonstrate a dynamical indicator that characterizes the onset of the glass transition in clusters and is consistent with other indicators of glass transitions in bulk systems. This criterion, based on changes in chaotic behavior as measured by the largest Liapunov exponent, reveals aspects of the microscopic processes associated with the phase change from liquid to glass, and provides a connection between the thermodynamic and dynamical behavior of systems and their multidimensional potential surfaces. (C) 1998 American Institute of Physics. [S0021-9606(98)51601-7]
\end{abstract}

When a liquid cools rapidly enough to avoid crystallization, ${ }^{1}$ it becomes a thermodynamically metastable fluid called a "supercooled" or "undercooled" liquid. With further cooling, the supercooled liquid transforms to an amorphous solid phase or a glassy state. ${ }^{2}$ The glassy state of matter, like the liquid state, has no long-range order but may have short-range order; unlike liquids, glasses have no capacity to flow. This investigation explores molecular phenomena underlying supercooling and glass formation and enables a detailed quantitative description of this process and of the glass transition in small finite systems, in a manner consistent with the bulk glass transition. One quantity of particular interest is the temperature at which the amorphous solid becomes unstable with respect to the corresponding crystal. $^{3}$ At this temperature, various measurable quantities, such as the heat capacity and viscosity, show discontinuities like those at the melting or freezing transition. ${ }^{2}$ Calorimetric and diffraction experiments, useful as they are, cannot probe microscopic changes near the transition temperature. At present, this understanding has to come from theoretical modeling and computer simulation. While plausible qualitative interpretations of dynamics and thermodynamics near the transition temperature are not difficult to find, generating a testable, quantitative description remains a formidable task. Part of this task is finding a suitable indicator to specify the phases and phase transition temperature of the system. Traditionally, the pair correlation function $g(r)$ has been used to characterize different phases through structural information: $g(r)$ shows a periodic order for crystalline solids, typically a split second peak for amorphous solids, and only broad peaks for liquids as a result of their short-range order. The utility of $g(r)$ seemed to diminish when it was found ${ }^{4}$ that the split in the second peak of $g(r)$ appears with supercooled liquids. This meant that the temperature dependence of $g(r)$ gives little or no information concerning phase changes of glass-forming materials. The Lindemann index ${ }^{5}$ is widely used as an indicator of melting or freezing for atomic systems but still needs a more precise definition to reach the same level of utility for molecular systems. ${ }^{6}$

Here we show how the changes among liquid, glass, and crystal, in both clusters and bulk materials, can be probed at the microscopic level through one particular dynamical index. Values we infer for this index imply that the amorphous solid may have a melting temperature below that of the melting point of the crystal. This is just the behavior to be expected if the amorphous solid is trapped in a metastable state with potential barriers lower than those associated with the deep, steep potential wells of the regular solid.

The time evolution of a system near a phase change may depend very sensitively on the initial phase-crystal, glass, liquid, or vapor; fluctuations leading to phase changes may involve extremely long relaxation or equilibrium times. Consequently, the limited intervals of simulations may become inadequate to reveal equilibrium phenomena. In such cases dynamical indicators of phase become particularly desirable ${ }^{7}$ to supplement geometric indicators. ${ }^{8}$ It is helpful to adopt a topographical interpretation, as advocated by Goldstein, ${ }^{9}$ Stillinger, ${ }^{10}$ and recently, by Kunz, Berry, Ball et al. ${ }^{11}$

At low temperatures, each system in an ensemble is confined to a single well, around a single minimum, on the potential surface. At slightly higher temperatures, each system may explore a basin containing several or many minima; with increasing energy or temperature, the volume of configuration space accessible to each system grows until the system passes into a region of a thermodynamically stable fluid state. The Lyapunov spectrum, the Kolmogorov entropy, and even just the largest positive Lyapunov exponent, are dynamical indicators of the way the system explores its phase space (and hence configuration space). Any sharp change in these quantities marks the onset of a transition of some kind.

The Lyapunov spectrum and maximum Lyapunov expo- 
nent (MLE) have been investigated for small Lennard-Jones and Morse clusters as measures of chaotic behavior (e.g., how strongly a locale on a potential surface drives trajectories apart) and of phase changes. ${ }^{12}$ Moreover, the evolution of the distribution of sample values of the MLE has proved to be an indicator of the development of ergodicity, ${ }^{13}$ an alternative to the (less readily accessible) fractal dimension of the trajectory in phase space. ${ }^{12}$ In this paper, we will demonstrate that the largest Lyapunov exponent characterizes structural transitions in somewhat larger clusters and in bulk material composed of rare-gas atoms.

The analysis is derived from constant-energy molecular dynamics simulations ${ }^{14}$ of the "magic-number'" clusters of 13 and 19 particles, and of a moderately large system of 108 particles. To model a bulk material, we applied periodic boundary conditions to the large cluster. Free boundary conditions were used with the smaller clusters. The potential is the sum of pairwise Lennard-Jones interactions, each of the form

$$
V\left(r_{i j}\right)=4 \epsilon\left(\left(\sigma / r_{i j}\right)^{12}-\left(\sigma / r_{i j}\right)^{6}\right),
$$

where $\epsilon$ is the unit of energy, $\sigma$ is the unit of length [in the sense that $V\left(r_{i j}\right)=-\epsilon$ when $\left.r=2{ }^{1 / 6} \sigma\right]$, and $r_{i j}$ is the distance between the atomic sites $i$ and $j$. The initial velocities are drawn randomly from a Maxwell-Boltzmann distribution. The velocity-Verlet algorithm served to integrate Hamilton's equations of motion under conditions of constant energy. ${ }^{14}$ The duration of the simulations at each temperature was approximately $1 \mathrm{~ns}$ of particle time. The boundary kept the density of the bulk system constant; the small, free clusters could of course expand, but did remain bound at the temperatures reported here. The average temperature, based on equipartition, is

$$
T=\frac{2 E_{\mathrm{kin}}}{(3 N-6) k_{b}},
$$

where $N$ is the number of atoms, $E_{\text {kin }}$ is the kinetic energy averaged over the entire trajectory, and $k_{b}$ is the Boltzmann constant, which we set equal to unity.

Melting in a bulk crystal was studied by starting the system from a regular fcc arrangement in which the particles are assigned small initial velocities. In contrast, the phase change in amorphous material is explored by starting from a frozen, random configuration obtained by quenching the high-energy liquid as follows. The system (whether bulk material or cluster) is heated to an energy high enough that its mean temperature puts it well in the liquid range. This ensures that the system is disordered and the atoms scramble among themselves. The system is then cooled at a high rate of the order of $10^{12} \mathrm{~K} / \mathrm{s}$ to yield a disordered but locally stable amorphous solid. Such quenching is known to yield amorphous structures for $\mathrm{Ar}_{19}$ (Ref. 11). The amorphous phase of the bulk material was characterized by its radial distribution function, $g(r)$; the amorphous structure of the cluster was analyzed by a detailed examination of the multidimensional potential energy surface and graphical visualization. Dynamical quantities such as mean squared displacement were computed for the quenched structures; when they were plotted as functions of time they were found to have slope zero, indicating the clusters were solidlike. The energies of the systems were then increased slowly by rescaling the velocities of the atoms by a factor slightly exceeding 1 .

We examined the properties of the trajectories, particularly their chaotic or regular character, by evaluating the maximum Lyapunov exponent, $\lambda$. This exponent measures the mean rate at which two trajectories diverge from each other, and may be defined as

$$
\lambda=\lim _{t \rightarrow \infty} \lim _{d(0) \rightarrow 0} \frac{1}{t} \ln \frac{d(t)}{d(0)},
$$

where $d(0)$ is the initial separation of two trajectories and $d(t)$ is their separation at time $t$. For regular motion, $\lambda=0$, and for chaotic motion $\lambda>0$. Here, with the conventional definition, $\lambda$ is a global average measure of the divergence and hence of the global average rate of growth, with length of trajectory, of the amount of information that must be retained in order to trace two trajectories back in time to see whether they were close at time $t=0$.

We have computed $\lambda$ using the tangent space method. ${ }^{15}$ To achieve convergence, we have used the following approach. At each temperature, the system was allowed to evolve for $3 \times 10^{5}$ molecular dynamics steps, and $\lambda$ was obtained from the final portion of the run. The average value of $\lambda$ evaluated over every 5000-step interval on the trajectory is essentially identical to the asymptotic value, which indicates that the limit process has effectively converged.

Figure 1(a) shows a typical pair distribution function $g(r)$, taken from one example of the confined 108 particles at a temperature $T=0.007$, in units of $\epsilon$. The first shell of neighbors is well defined and falls at a separation distance of $r=1.12 \sigma$. The next double peak, with maxima at $r=1.93$ and $2.16 \sigma$, implies by its split shape, an absence of longrange order, which may be taken as a signature of an amorphous structure.

Figure 2 shows the variation of $\lambda$ for the 108-particle caged cluster as a function of temperature, for both the quenched, amorphous form (diamond points) and the wellannealed crystal (crosses). At low mean temperatures, the system is confined to vibrate in a single potential well, whether it is amorphous or crystalline; because the well for the crystal is deeper and steeper than those for the random amorphous forms, the values of $\lambda$ at low temperatures are somewhat higher for the amorphous form, whose motions are more anharmonic than those of the crystal. Then, as the energy is increased, the values of $\lambda$ increase for both crystal and amorphous forms, but faster for the latter. This is presumably related to the way the densities of states of nonrigid, amorphous systems increase more rapidly with energy than do those of more rigid, ordered solidlike systems. ${ }^{16}$ At $T$ $=1.22, \lambda$ increases sharply for the amorphous form but not for the crystal. At this temperature, the amorphous form appears to melt. This is a sharp, single temperature because the periodic boundary conditions make this behave like a bulk system. It appears that the sharp rise in the $\lambda$ is an index of melting, in contrast to its behavior for extremely small sys- 


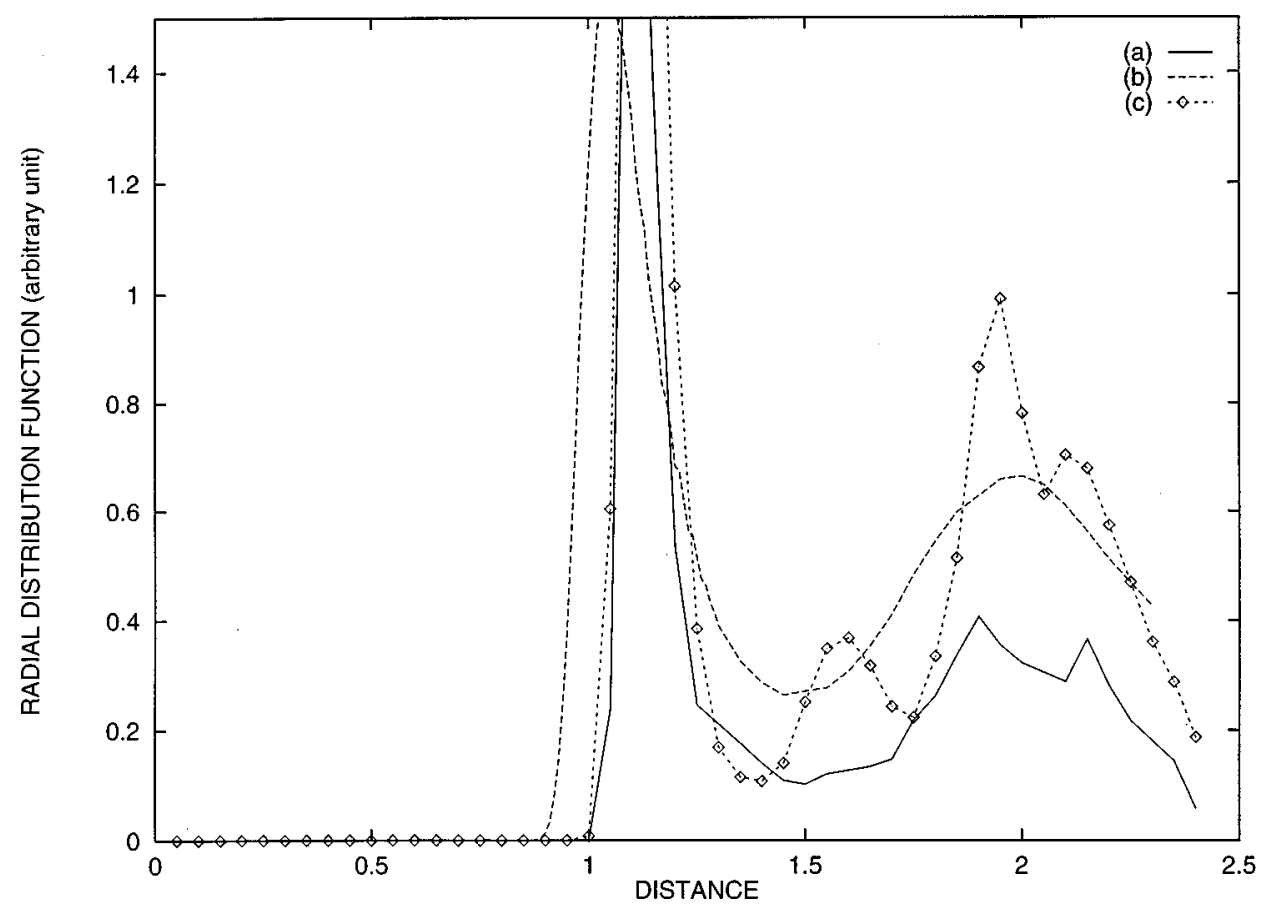

FIG. 1. Plot of the radial distribution function, $g(r)$ of 108 particles with periodic boundary conditions at (a) $T=0.01$, implying the amorphous structure of the bulk material, (b) $T=1.81$, indicating the liquid character of the system, (c) $T=0.11$, confirming the triggering in amorphous solid (see Fig. 2 and text for details).

tems, in which $\lambda$ drops at energies at which the system can just cross the saddles of the soft vibrational mode and enter large-amplitude motions. ${ }^{12}$ As systems grow larger, their soft modes comprise a smaller and smaller fraction of the total, and their saddles seem to become sharper, so that the chaotic character of all the other modes overshadows any low kinetic energy and tendency toward regular behavior associated with long intervals in saddle regions. ${ }^{17}$ The Kolmogorov entropy of Lennard-Jones clusters of 7 particles shows no detectable change with increasing energy, passing from solid to solid-

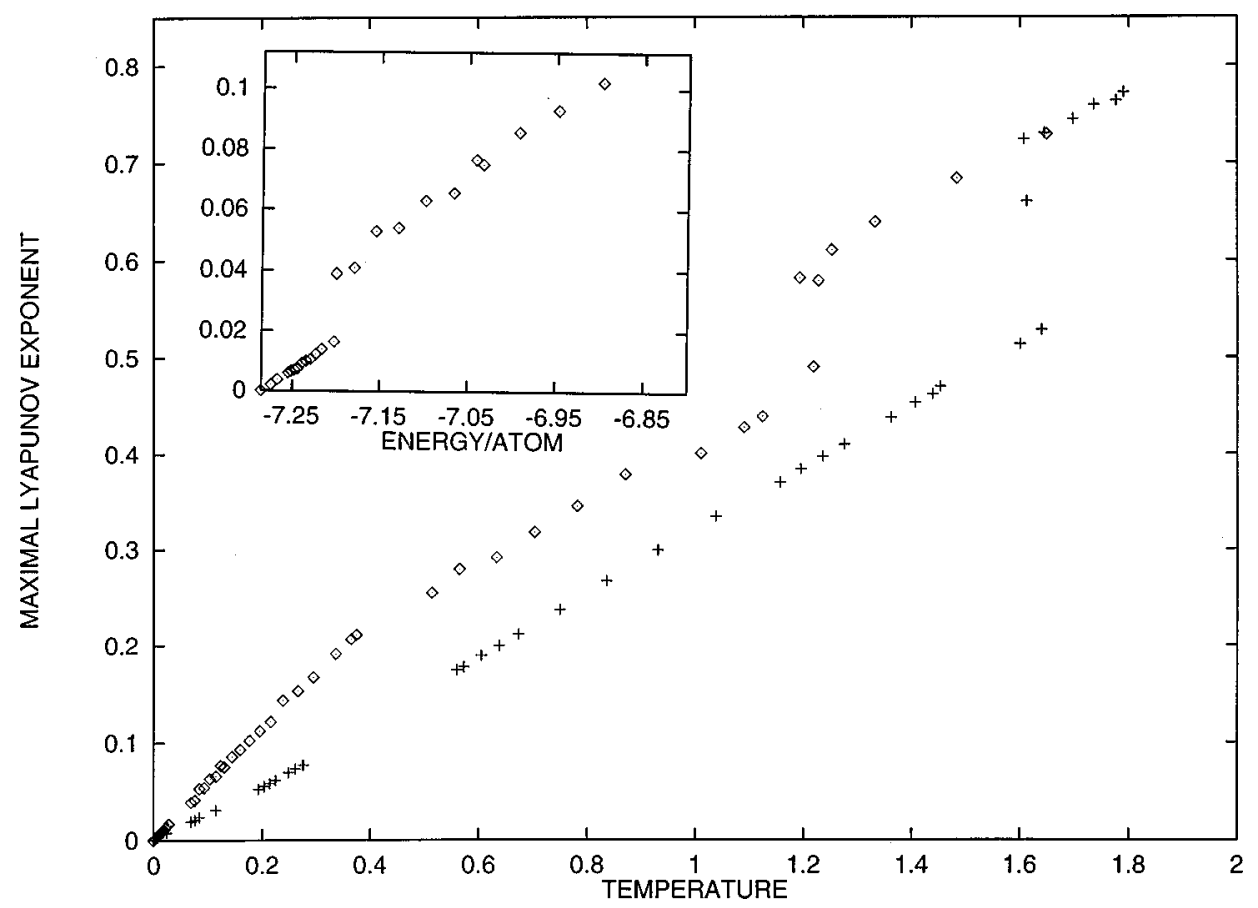

FIG. 2. Graph showing the behavior of MLE with temperature for crystalline solid (+) and amorphous material (diamond symbol). The amorphous solid melts at a lower temperature than the corresponding crystal. The insert shows a variation of MLE versus energy/atom of an amorphous system in an expanded scale in the temperature range between 0.01 and 0.55 . The sudden jump of MLE at $-7.20 \epsilon /$ atom (or $T=0.1$ ) corresponds to crystallization in the system. 
liquid co-existence to liquid. ${ }^{12}$ The 108-particle amorphous system simulating bulk material has the intuitively expected behavior, a sharp increase in chaotic character at the point of melting of the glass. At sufficiently low temperatures, the amorphous material is trapped in a single minimum of its multidimensional potential surface and vibrates approximately harmonically, so its Lyapunov exponents go to zero.

The crystal, by comparison, exhibits stronger binding, and consequently a larger heat of fusion than that of the amorphous material. The MLE of the crystal has an analogous sharp rise, but at a higher temperature, 1.61. ${ }^{18}$ Moreover, the crystal's MLE has a jump larger than that of the amorphous material, indicating a greater change in the degree of chaotic behavior. If the entropy of the crystal, in contrast to the Kolmogorov entropy, were much lower than that of the amorphous solid, then, because of the difference in the temperatures of the breaks in the two MLE curves, both of which we interpret as characterizing melting points, could have similar heights, because of the large differences in their heats of fusion. That they are moderately far apart is a measure of some degree of similarity of the entropies of the two forms of solid. At low temperatures, the entropy and the Lyapunov exponents of the crystal go to zero, but the crystalline form is more regular and has lower values of $\lambda$ than the amorphous form. Presumably some low barriers on the potential surface of the amorphous system allow motion at temperatures at which the atoms of the crystal have virtually lost all their internal mobility. The Lyapunov exponents of the amorphous material do go to zero at sufficiently low temperatures; presumably the vibrational entropy does also, but there can be some ambiguity regarding the configurational entropy and the meaning of very low temperatures for the amorphous material. ${ }^{19}$

The interpretation of the break as arising from melting is supported by the form of the pair distribution function $g(r)$ just above this transition region, as shown in Fig. 1(b). The split second peak becomes a single, broad peak, thought to be characteristic of the transition to a fluid state. Supercooled liquids also may show such split peaks, ${ }^{4}$ especially in mixtures, so we can infer from collapse of the doubled second peak in $g(r)$ that the system forms a normal, equilibrated fluid there. A comparable index for defining the glass transition, especially in mixtures, would be very useful. ${ }^{20}$

The insert of Fig. 2 shows the low-temperature region of the curve of $\lambda$ for the amorphous material. The break at $T$ $=0.07$ or an energy of $-7.20 \epsilon$ per atom occurs at the transition between the amorphous and crystalline solids. Figure 1(c) establishes this with the behavior of $g(r)$ just above the transition point. A new peak at $r=\sqrt{2} \sigma$ starts to appear and the second peak in the doublet disappears. Because of the problem of long time scales mentioned in the introduction, this is more likely to be a phenomenon associated with cooling of the amorphous material below a limit of its metastability than a true first-order phase transition. This break occurs in the amorphous system, not in the crystal. This may be analogous to a phenomenon seen in protein dynamics ${ }^{21}$ and in $\mathrm{Si}^{22}$ However, there is some uncertainty regarding the crystallization temperature that converts amorphous to crys- tallized Si. Computer simulations could be designed to give insight into and possibly resolve this question. ${ }^{23}$

Many small clusters may show changes that are counterparts of bulk, first-order phase transitions, and merge into first-order transitions as the clusters become larger. ${ }^{24}$ The cluster behavior, however, is strikingly different from a bulk phase transition, particularly for clusters of a few hundred particles or less. Specifically, two or more phaselike forms of clusters may coexist in bands of temperature and pressure, like a bulk system's components (rather than phases), in dynamic equilibrium. The causes can be lumped into "finite size effects,' which include a variety of factors such as the small differences in free energies of the various phaselike forms, the large number of particles on the surface, and the absence of any constraint requiring periodicity. Because it is possible to follow the behavior of clusters as the number $N$ of component particles becomes very large, one can use small systems to interpret many of the important characteristics of larger systems, such as the topographical character, in terms of the linked sequences of minima and saddles. ${ }^{11}$

Here we have investigated the behavior of the maximum Lyapunov exponent of the 19-atom Lennard-Jones cluster in two ways: we have examined the energy dependence of the exponent itself, and we have studied the distribution of the sample values from which the exponent is inferred. We have studied the 13-particle cluster as well, and find similar conclusions. The temperature dependence of $\lambda$ for the 19 -atom cluster is shown in Fig. 3. At low temperature, $\lambda$ is zero or a very small number, and increases with the increase of temperature, showing a sharp rise at an energy of approximately $-3.25 \epsilon$ per atom corresponding to $T=0.25$ in the same units of the Lennard-Jones parameter. The scattered values of $\lambda$ in this region are due to the multiphase dynamical equilibrium in which the system explores the different phases (solidlike and liquidlike), and consequently different regions in the phase space. The distributions of sample values of $\lambda(5000$ steps) at these energies are bimodal, separating into regions of high and low chaotic character, consistent with earlier studies of smaller systems. Figure 4 shows such distributions for the 13-particle cluster at three energies: one in the coexistence region, one below, and one above. In the coexistence region, the highly chaotic part of the distribution with the larger $\lambda$ corresponds to the high-kinetic-energy region and to an energetic, solidlike state of the dynamically coexistence phases; the part of the distribution showing relatively low chaotic character and a smaller range of values for the $\lambda$ corresponds to a region of low kinetic energy and to the liquidlike partner of the coexisting phases.

A plot of $\lambda$ versus energy in the low temperature region is shown in the inset of Fig. 3. In this region, the sharp break at $T=0.17$ corresponds to the temperature at which the amorphous structure becomes unstable. Unlike in bulk, there is no particular change in $g(r)$ at this energy. This behavior of the amorphous cluster is quite similar to the transition between the amorphous and crystalline phases in bulk material (see Fig. 2) and can be taken as an indication of glassy behavior in the small cluster. However, the occurrence of bands of solid-liquid coexistence in these systems imposes 


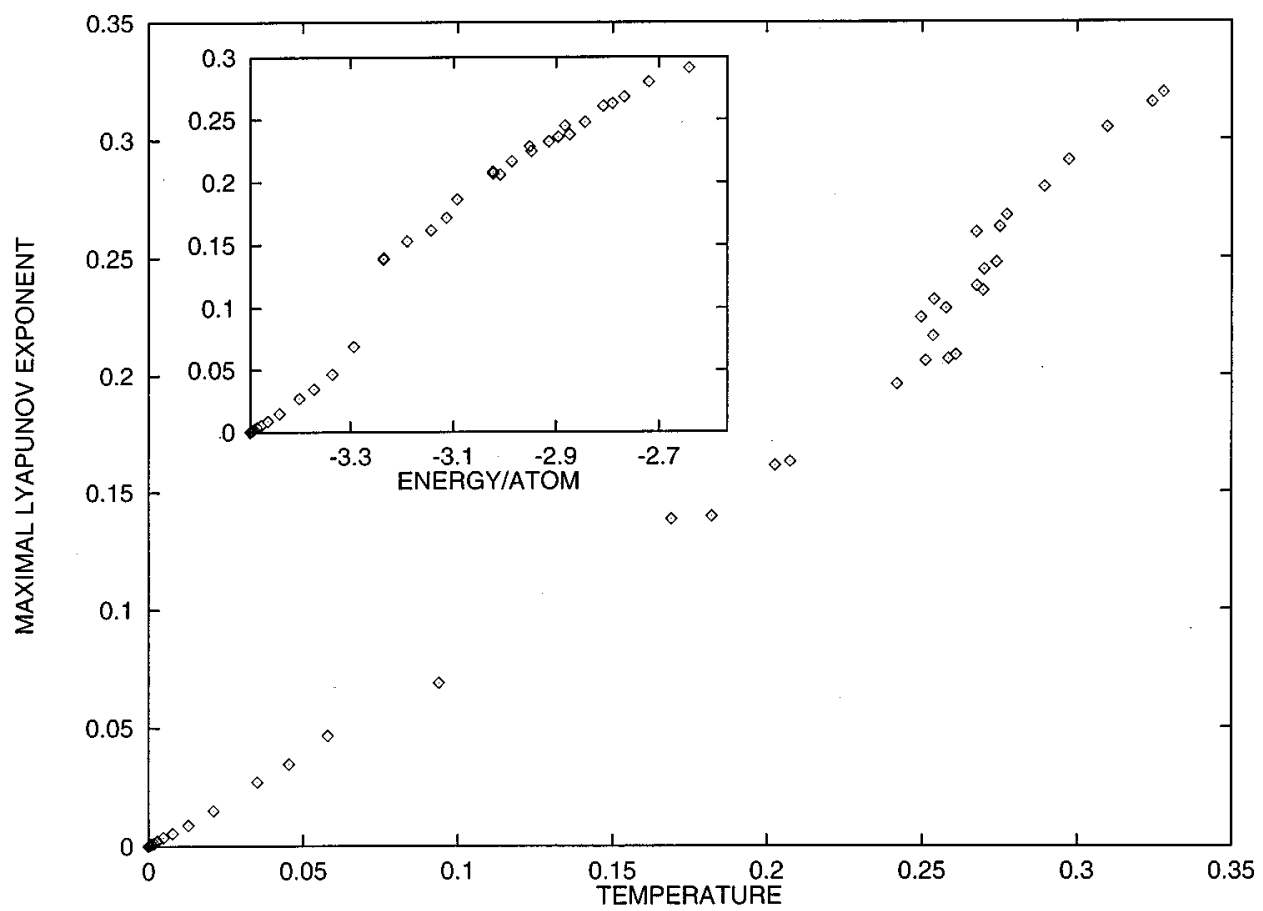

FIG. 3. Plot of MLE versus temperature for $\mathrm{Ar}_{19}$. The insert shows a variation of MLE versus energy in an expanded scale.

an imprecision on the identification of distinct melting regions for crystalline and amorphous forms of the finite solid, at least in this size range. Simulations in larger free clusters could possibly resolve the two melting zones, as they sharpen to points.

The results presented here introduce the largest Lyapunov exponent and the distribution of its sample values as indices to characterize phase transitions, in both bulk matter and clusters. The sharp change in the value of this exponent in both moderate-size clusters and in simulated bulk matter is consistent with the entropy change accompanying a first-order phase change or its finite-system equivalent. Direct comparison of the structural entropy and distributions of phase space ${ }^{25}$ near the transition temperature where $\lambda$ shows

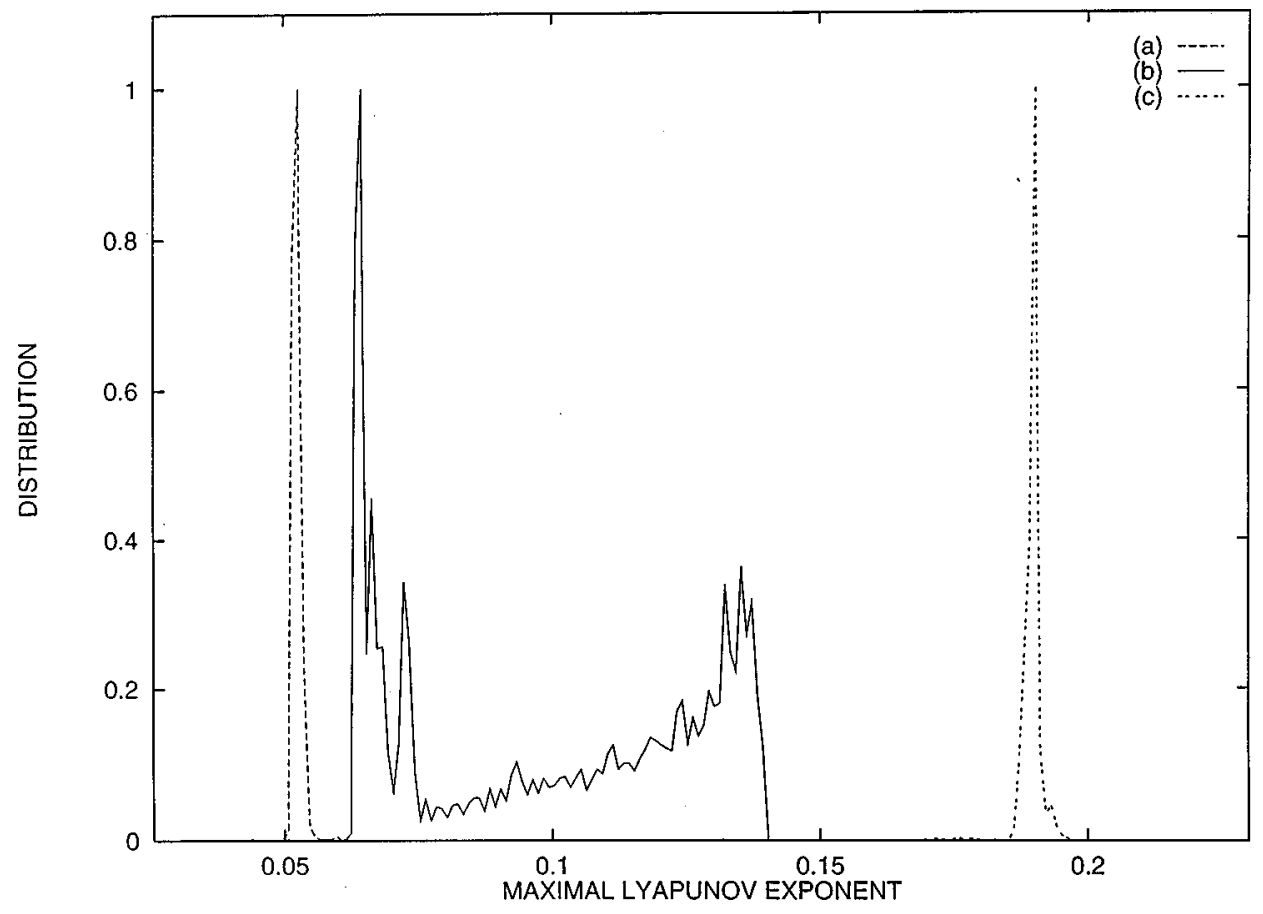

FIG. 4. Distributions of MLE for $\operatorname{Ar}_{13}$ (a) below the coexistence region, $E=-3.18 \epsilon /$ atom, (b) in the coexistence region, $E=-2.78 \epsilon /$ atom, (c) above the coexistence region, $E=-2.72 \epsilon /$ atom (see the text for details). 
a sharp jump will be published elsewhere. It will be useful if diffraction $^{21}$ or heat capacity measurements ${ }^{26}$ can be carried out to probe the sharp change found here in the Lyapunov exponents. Confirmation would allow us to add the dynamical concept of increasing Kolmogorov entropy, a time rate of change of entropy, to the changes of thermodynamic properties - energy, entropy, and density - that we associate with the melting transition.

\section{ACKNOWLEDGMENTS}

Two of us (S. K. N. and P. J.) would like to acknowledge the financial support from the Department of Energy. S. K. N. would like to thank Professor R. Ramaswamy for helpful discussions. K. D. B. and R. S. B. would like to acknowledge support from the National Science Foundation for their contributions to this research.

${ }^{1}$ C. A. Angell, Science 267, 1924 (1995).

${ }^{2}$ J. Wong and C. A. Angell, Glass Structure by Spectroscopy (Marcel Dekker, New York, 1976).

${ }^{3}$ F. H. Stillinger, Science 267, 1935 (1995).

${ }^{4}$ H. Jonson and H. C. Andersen, Phys. Rev. Lett. 60, 2295 (1988).

${ }^{5}$ F. A. Lindemann, Phys. Z. 11, 609 (1910); I. Z. Fisher, Statistical Theory of Liquids (University of Chicago Press, Chicago, 1966).

${ }^{6}$ L. S. Bartell, F. J. Dulles, and B. Chuko, J. Phys. Chem. 95, 6481 (1991).

${ }^{7}$ P. Brumer, Adv. Chem. Phys. 47, 201 (1981).

${ }^{8}$ R. A. Laviolette and F. H. Stillinger, J. Chem. Phys. 83, 4079 (1985).

${ }^{9}$ M. Goldstein, J. Chem. Phys. 51, 3728 (1969).
${ }^{10}$ F. H. Stillinger, J. Chem. Phys. 88, 7818 (1988); F. H. Stillinger and T. A. Weber, Science 225, 983 (1984).

${ }^{11}$ R. E. Kunz and R. S. Berry, J. Chem. Phys. 103, 1904 (1995); R. S. Berry and R. E. Kunz, Phys. Rev. Lett. 74, 3951 (1995); K. D. Ball, R. S. Berry, R. E. Kunz, F. Li, A. Proykova, and D. J. Wales, Science 271, 963 (1996).

${ }^{12}$ T. L. Beck, D. M. Leitner, and R. S. Berry, J. Chem. Phys. 89, 1681 (1988); D. Leitner, R. S. Berry, and R. M. Whitnell, ibid. 91, 3470 (1989); R. J. Hinde, R. S. Berry, and D. J. Wales, ibid. 96, 1376 (1992); S. K. Nayak, R. Ramaswamy, and C. Chakravarty, Phys. Rev. E 51, 3376 (1995)

${ }^{13}$ C. Amitrano and R. S. Berry, Phys. Rev. Lett. 68, 729 (1992); Phys. Rev. E 47, 3158 (1993).

${ }^{14}$ M. P. Allen and D. J. Tildesley, Computer Simulation of Liquids (Oxford University Press, Oxford, 1987).

${ }^{15}$ G. Benettin, L. Galgani, and J. M. Strelcyn, Phys. Rev. A 14, 2338 (1976).

${ }^{16}$ R. S. Berry, J. Jellinek, and G. Natanson, Phys. Rev. A 30, 919 (1984); Chem. Phys. Lett. 107, 227 (1984).

${ }^{17}$ R. J. Hinde and R. S. Berry, J. Chem. Phys. 99, 2942 (1993).

${ }^{18}$ A. Rahman, M. J. Mandell, and J. P. McTague, J. Chem. Phys. 64, 1564 (1976).

${ }^{19}$ W. Kauzmann, Chem. Rev. 43, 219 (1948)

${ }^{20}$ S. K. Nayak and R. Ramaswamy, J. Phys. Chem. 98, 9260 (1994), S. K. Nayak (unpublished).

${ }^{21}$ W. Doster, S. Cusack, and W. Petry, Nature (London) 337, 754 (1989); B. F. Rasmussen, A. M. Stock, D. Ringe, and G. A. Petsko, ibid. 357, 423 (1992).

${ }^{22}$ M. O. Thomson, G. J. Galvin, J. M. Mayer, P. S. Peercy, J. M. Poate, D. C. Jacobson, and A. G. Cullis, Phys. Rev. Lett. 52, 2360 (1984).

${ }^{23}$ P. Baeri, Phys. Rev. Lett. 45, 2036 (1980); S. A. Kokorowski, et al. ibid. 48, 498 (1982).

${ }^{24}$ R. S. Berry, Chem. Rev. 93, 2379 (1993).

${ }^{25}$ C. Seko and K. Takatsuka, J. Chem. Phys. 104, 8613 (1996).

${ }^{26}$ C. T. Moynihan, Ann. (N.Y.) Acad. Sci. 279, 15 (1976). 